\title{
Molecular Characterization, Phylogenetic Relationships, and Specific Detection of Peach mosaic virus
}

\author{
D. James, A. Varga, H. Croft, H. Rast, D. Thompson, and S. Hayes
}

Sidney Laboratory, Canadian Food Inspection Agency, 8801 East Saanich Road, Sidney, B.C., Canada, V8L 1H3. Accepted for publication 14 September 2005.

\begin{abstract}
James, D., Varga, A., Croft, H., Rast, H., Thompson, D., and Hayes, S. 2006. Molecular characterization, phylogenetic relationships, and specific detection of Peach mosaic virus. Phytopathology 96:137-144.

Peach mosaic virus (PcMV) and Cherry mottle leaf virus (CMLV) are serologically related viruses that cause distinct diseases, have a different host range, and are vectored by different eriophyid mites. Sequence analysis of the genome of PcMV indicates that it is closely related genetically to CMLV but distinct, with similar genome organization and a member of the genus Trichovirus. The genome of PcMV consists of 7,988 nucleotides, excluding a poly(A) tail at the $3^{\prime}$ end of the genome. Four putative open reading frames (ORF1 to 4) were identified coding for proteins of $216.3,47.2,21.7$, and $15.7 \mathrm{kDa}$, respectively. Also, three noncoding regions were identified, including an intergenic region separating ORF3 and ORF4. The complete nucleotide sequence of PcMV shares

isolates of PcMV ranged from 97 to $99 \%$ versus $83 \%$ identity when compared with the CP of CMLV. In vitro expression and subsequent western blot analysis confirmed ORF3 as encoding the CP gene of PcMV. Phylogenetic analysis supports classification of PcMV and CMLV as members of the genus Trichovirus. They are unique members of this genus with an extra ORF (ORF4). PcMV ORF4 appears to code for a putative nucleic acid-binding (NB) protein which has identity with the NB protein of CMLV and members of the genera Allexivirus, Carlavirus, and Vitivirus. PcMV and CMLV appear to be the products of recombination between members of the genus Trichovirus and a virus group containing the putative NB protein. Alternatively, PcMV and CMLV may represent the intact genome, with a deletion event producing members that lack ORF4. A reverse transcription-polymerase chain reaction procedure was developed for reliable and specific detection of PcMV. This will be an asset for stone fruit virus certification.
\end{abstract} 73\% identity with CMLV. The CP amino acid sequence identity between
Peach mosaic is an important disease of stone fruits causing symptoms that may include retarded foliation, mottling and deformity of leaves, fruit deformation, and stunted plant growth $(21,23)$. The disease has the potential to cause severe economic losses since it affects several commercially cultivated species including almond (Prunus dulcis (Mill.) D.A. Webb), apricot (P. armeniaca L.), peach (P. persica (L.) Batsch), and plum (P. domestica L.) (23). Peach mosaic was first described in 1931 in Texas and Colorado $(2,11)$. The disease spreads rapidly $(7$ infected trees in Colorado in 1931 versus 30,467 trees in 1935) and produces severely deformed fruit that are unmarketable $(23,26)$. Peach mosaic has been reported to occur in Mexico and the United States $(23,25,26)$, also in Greece, Italy, and India (23). It is of economic importance in western Colorado and southern California, in the United States (26).

The causal agent was identified as a flexuous virus (Peach mosaic virus, PcMV), 752 to $888 \mathrm{~nm}$ long, $9.25 \mathrm{~nm}$ wide, and with striations $3.47 \mathrm{~nm}$ in pitch $(7,13)$. The coat protein $(\mathrm{CP})$ subunit molecular mass was estimated by western blot analysis at $20.5 \mathrm{kDa}$, with a genome size estimated at 8.1 to $8.2 \mathrm{~kb}(7,12,13,15)$.

There is a close serological relationship between PcMV and Cherry mottle leaf virus (CMLV). Polyclonal and/or monoclonal antibodies derived from either virus react with the heterologous virus $(7,12,13,25)$, and both viruses have $\mathrm{CP}$ subunits estimated at $20.5 \mathrm{kDa}(12,13)$. Both viruses are transmitted by eriophyid mites but by different species. PcMV is transmitted by Eriophyes insidiosus Keifer and Wilson (19), while CMLV is transmitted by

Corresponding author: D. James; E-mail address: jamesd@ inspection.gc.ca

DOI: $10.1094 /$ PHYTO-96-0137

This article is in the public domain and not copyrightable. It may be freely reprinted with customary crediting of the source. The American Phytopathological Society, 2006.
E. inaequalis Wilson and Oldfield (24). There are differences in the host range and the diseases associated with the two viruses. CMLV causes severe symptoms on susceptible cherry cultivars, while PcMV is not known to affect cherry (23). P. avium (L.) 'Bing' is used as a woody indicator for CMLV infection, displaying symptoms of irregular chlorotic mottling and distortion of the leaves $(15,23)$. 'Bing' cherry is not a host for PcMV (13).

Traditionally, PcMV has been detected by indexing on the woody indicator $P$. persica cv. Elberta (23). More recently, serological and nucleic acid-based tests have been developed for the detection of PcMV $(7,13,16,25)$. These include a reverse transcription-polymerase chain reaction (RT-PCR) test targeting the replicase gene of PcMV, with amplification of a 419-bp fragment (16). In evaluating the specificity of the PcMV primers, nonspecific amplification was observed with some isolates of Apple chlorotic leaf spot virus (ACLSV). ACLSV is the type member of the genus Trichovirus, family Flexiviridae (1). CMLV has been sequenced (14), and analysis of the genome has resulted in the classification of the virus as a definitive member of the genus Trichovirus (1). PcMV is classified as a member of the genus Trichovirus (1) based on its relationship to CMLV and the limited sequence data available. Therefore, PcMV is closely related to ACLSV.

In this study, sequencing of the genome of PcMV was undertaken with the objectives of (i) determining the relationship of PcMV to CMLV at the molecular level, (ii) obtaining information that would allow definitive classification of the virus, and (iii) identifying sequences of PcMV that might be suitable for designing oligonucleotide primers for use in PcMV-specific RT-PCR, for reliable identification and control of this virus. The results obtained support the classification of PcMV as a member of the genus Trichovirus, and indicate a close but distinct relationship with CMLV. PcMV and CMLV appear to be the products of recombi- 
nation between members of the genus Trichovirus and members of a nucleic acid-binding protein-containing group of viruses, likely within the family Flexiviridae. Also, a PcMV-specific RTPCR procedure was developed that showed no signs of cross reaction with a broad range of viruses belonging to the family Flexiviridae.

\section{MATERIALS AND METHODS}

Virus sources. PcMV isolate CA-1 (2022-01) was maintained in peach and in the herbaceous host Chenopodium quinoa. Partial sequence data was generated using PcMV CA-1 (16) so this isolate was selected for sequencing. Isolates CA-2 (2022-02), CA-3 (2022-03), and CL-2 (2022-04) were maintained in peach, and the $\mathrm{CP}$ region of each isolate was sequenced using RNA extracted directly from the peach host. These isolates are part of a virus collection maintained in secure screenhouses at the Sidney Laboratory. All PcMV isolates were provided by W. E. Howell and H. J. Larsen (13). Table 1 provides a complete list of viruses included in this study.

Double-stranded RNA extraction and cDNA synthesis. PcMV isolate CA-1 (2022-01) was propagated in the herbaceous indicator plant $C$. quinoa and used as the source for the isolation of PcMV double-stranded RNA (dsRNA). dsRNA was extracted using a procedure similar to that described by Morris and Dodds (22). A gel-purified $7.0 \times 10^{6} \mathrm{Da}$ dsRNA fragment, presumed to represent the complete genome of PcMV $(7,13)$, was used as the template for cDNA synthesis. The dsRNA was denatured by heating at $94^{\circ} \mathrm{C}$ for $10 \mathrm{~min}$ prior to reverse transcription (16). Total RNA was used in some cases where PcMV-specific primers were available such as for generating the $\mathrm{CP}$ sequences of the various PcMV isolates. The $3^{\prime}$ end of the genome was generated using an oligo(dT) primer combined with random primers, since earlier studies by James and Upton (16) indicated that PcMV was polyadenylated at the $3^{\prime}$ terminus. The rest of the genome was generated by combining virus-specific antisense primers with random primers, and using the purified $7.0 \times 10^{6} \mathrm{Da}$ dsRNA fragment as a template in RT-PCR. The $5^{\prime}$ terminus of the virus was generated using Invitrogen's 5' RACE System for Rapid Amplification of cDNA ends, version 2.0 (\#18374-058, Invitrogen, Burlington, Ontario).

Cloning of cDNA products. Amplified cDNA fragments were identified by agarose (1\%) gel electrophoresis with ethidium bro- mide staining, excised from the agarose gel, and purified using Qiagen's MiniElute Gel Extraction Kit (\#28604, Qiagen, Mississauga, Ontario). The PCR fragments were generated using Taq DNA polymerase. Cloning was carried out using the TOPO TA Cloning Kit, as directed by the supplier (Invitrogen, Carlsbad, CA). Taq Extender PCR Additive (Stratagene, La Jolla, CA) was combined with Taq DNA polymerase to improve reaction fidelity.

Sequencing and sequence analysis. Sequencing and sequence analysis were carried out as described by James and Varga (17).

Expression of the PcMV CP. Sequences encoding the CP gene of PcMV were amplified using the forward primer PcMCP-F (5'-GAGCTCATGTCGGCAAGATTGAAC-3') with a SacI site attached (underlined) and the reverse primer PcMCP-R (5'AAGCTTCTAAACACACAAATTC-3') with a HindIII site attached (underlined). The primers were designed to facilitate insertion of the PcMV putative $\mathrm{CP}$ derived cDNA into the SacI/HindIII sites of pCOLD I expression vector (Takara Mirus Bio Corp., Madison, WI). The primers amplify a 591-bp fragment which was gel purified (1.5\% agarose, QIAquick Gel Extraction Kit, Qiagen), ligated into the pCR 2.1 TOPO vector, and cloned using the TOPO TA Cloning Kit (Invitrogen). The 591-bp insert was digested with $\mathrm{SacI} / \mathrm{HindIII}$ and ligated into the corresponding restriction site of pCOLD I. The pCOLD vector uses the Cold Shock Protein A ( $\operatorname{csp} \mathrm{A})$ promoter. The vector contains a translation enhancing element (TEE), a His-Tag sequence, and Factor Xa site which are incorporated in the expressed recombinant or fusion protein. A single colony of Escherichia coli BL21 (DE3) transformed with pCOLD/PcM-CP (vector with insert) was used to inoculate a 5-ml culture of Luria-Bertani (LB) broth and ampicillin $(100 \mu \mathrm{g} / \mathrm{ml})$, which was incubated overnight $(16 \mathrm{~h})$ at $37^{\circ} \mathrm{C}$ with shaking at $250 \mathrm{rpm}$. A $50-\mathrm{ml} \mathrm{LB}$ culture was inoculated with $200 \mu \mathrm{l}$ of the overnight culture and incubated at $37^{\circ} \mathrm{C}$ with shaking at $250 \mathrm{rpm}$, until $A_{600}=0.500$ was reached. The culture was equilibrated to $15^{\circ} \mathrm{C}$ and induced with $1 \mathrm{mM}$ IPTG for $24 \mathrm{~h}$. The induced culture was pelleted at 7,500 rpm for $5 \mathrm{~min}$, resuspended in $10 \mathrm{ml}$ of urea binding buffer $(8 \mathrm{M}$ urea, $100 \mathrm{mM}$ $\mathrm{NaH}_{2} \mathrm{PO}_{4}, 10 \mathrm{mM}$ Tris, $\mathrm{pH} 8.0$ ), sonicated $5 \times 10 \mathrm{~s}$, and centrifuged at 10,000 rpm for $10 \mathrm{~min}$. The supernatant was mixed with $100 \mu \mathrm{l} \mathrm{Ni-NTA}$ agarose (Qiagen) for $30 \mathrm{~min}$ at room temperature, and then packed into a column and washed twice with $10 \mathrm{ml}$ of urea wash buffer ( $8 \mathrm{M}$ urea, $100 \mathrm{mM} \mathrm{NaH} \mathrm{PO}_{4}, 10 \mathrm{mM}$ Tris, $\mathrm{pH}$ 6.3). The fusion PcMV CP was eluted with $4 \times 200 \mu \mathrm{l}$ urea elution buffer ( $8 \mathrm{M}$ urea, $100 \mathrm{mM} \mathrm{NaH} \mathrm{PO}_{4}, 10 \mathrm{mM}$ Tris, $\mathrm{pH} 4.5$ ).

TABLE 1. Virus isolates screened by reverse transcription-polymerase chain reaction (RT-PCR) using Peach mosaic virus (PcMV)-derived primers (A and B)

\begin{tabular}{|c|c|c|c|c|}
\hline Accession & Host & Disease status $^{\mathrm{a}}$ & RT-PCR $(\mathrm{A})^{\mathrm{b}}$ & RT-PCR $(B)^{b}$ \\
\hline 2022-01(CA1) & Peach & PcMV & + & + \\
\hline 2022-02(CA2) & Peach & PcMV & + & + \\
\hline 2022-03(CA3) & Peach & PcMV & + & + \\
\hline 2022-04(CL2) & Peach & PcMV, PLMVd & + & + \\
\hline $1162-21$ & Cherry & CMLV, CGRMV, CVA, PNRSV & - & - \\
\hline $1819-02$ & Cherry & CMLV, CGRMV, CVA, PNRSV, PDV, ApGUM & - & - \\
\hline 38567 & Peach & ACLSV, CGRMV, PLMVd & + & - \\
\hline $982-11$ & Apple & ACLSV, ASPV, ASGV, ARW, AFL & + & - \\
\hline $996-1$ & Plum & ACLSV & + & - \\
\hline $2228-03$ & Apple & ACLSV, CFD & - & - \\
\hline $1000-04$ & Apple & ACLSV & - & - \\
\hline $70-08$ & Apple & ACLSV, ASPV, ARW & - & nt \\
\hline 38686 & Almond & ACLSV & - & $\mathrm{nt}$ \\
\hline $1624-01$ & Pear & ACLSV, ASPV & - & nt \\
\hline $340-02$ & Cherry & CGRMV, CVA, LCHV-2 & - & $\mathrm{nt}$ \\
\hline $1586-17$ & Grapevine & GVA & - & nt \\
\hline $3138-02$ & Grapevine & GVB & - & nt \\
\hline $3138-06$ & Grapevine & GVA, GVB & - & nt \\
\hline
\end{tabular}

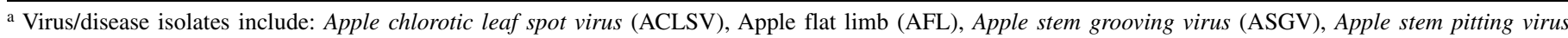
(ASPV), Apple rubbery wood (ARW), Apricot gummosis (ApGUM), Chat fruit disease (CFD), Cherry green ring mottle virus (CGRMV), Cherry mottle leaf virus (CMLV), Cherry virus A (CVA), Grapevine virus A (GVA), Grapevine virus B (GVB), Little cherry virus-2 (LCHV-2), Prune dwarf virus (PDV), Peach latent mosaic viroid (PLMVd), and Prunus necrotic ring spot virus (PNRSV).

${ }^{b}$ RT-PCR(A) with primers PM16AFF/16AFR. RT-PCR(B) with primers PM-AF1/PM-AFR. $+=$ positive, $-=$ negative. 
Western blot analysis was carried out using the monoclonal antibody (MAb) 1162-A2 as described by James and Howell (13).

Total RNA extraction. Total RNA was extracted from healthy and infected herbaceous and woody plants using the approach described by James et al. (18).

Development of PcMV-specific RT-PCR. RT-PCR was carried out according to the procedure described by James and Upton (16). This RT-PCR, utilizing PcMV-derived primers PM16AFF and PM16AFR (16), was used to screen a range of virus isolates representing different genera within the family Flexiviridae (Table 1). In evaluating the specificity of this assay, cross reactions were observed with some isolates of ACLSV (Table 1). Once the complete genome sequence of PcMV was determined, the sequence was analyzed and compared with ACLSV and CMLV sequences in GenBank. The primers PM16AFF and PM16AFR target the $3^{\prime}$ terminus of the coding region of the replication protein (open reading frame 1 [ORF1]), and amplify a fragment of 419 bp (16). RT-PCR with these primers is identified in this study as RT-PCR(A). The replicase region was identified as a suitable target region, and a series of new primers were designed, tested for their efficiency in RT-PCR, and evaluated for specific detection of PcMV. The most effective pair of new primers was forward primer PM-AF1 (5'-TCACCTTCTGCAGATACGAAGTA-3', nucleotide 5292-5314) and reverse primer PM-AFR (5'GCTGTTCTTCACAAAGAATCTA-3', nucleotide 5653-5674). These primers amplify a 383-bp fragment at the $3^{\prime}$ end of the replicase coding region, and assays utilizing these primers are identified in this study as RT-PCR(B).

\section{RESULTS}

Sequence assembly and genome organization. Approximately 40 plasmids with cDNA inserts, generated by RT-PCR, were used to determine the sequence of PcMV with at least two plasmids covering each region. In most cases, sequence accuracy was improved by sequencing both strands of the cDNA insert. The genome of PcMV consists of 7,988 nucleotides (nts), excluding the poly(A) tail at the $3^{\prime}$ end of the genome. Four putative ORFs were identified coding for proteins with molecular masses of $216.3 \mathrm{kDa}(1,886$ amino acids [aa]), $47.2 \mathrm{kDa}$ (422 aa), 21.7 kDa (193 aa), and $15.7 \mathrm{kDa}(127 \mathrm{aa})$, from ORF1 to ORF4, respectively (Fig. 1). Three noncoding regions (NCRs) were identified: (i) at the $5^{\prime}$ end, (ii) between ORF3 and ORF4, and (iii) at the 3' end of the genome, upstream of the poly(A) tail. ORF1, ORF2, and ORF3 overlap each other, with a 148-nt NCR separating ORF3 from ORF4. BLAST searches (National Centre for Biotechnology Information, National Institutes of Health) and pairwise comparisons showed that the PcMV sequence had maximum identity to CMLV, ranging from 51 to $86 \%$ for the various regions of the virus (Table 2). The genome organization of PcMV is identical to that of CMLV as described by James et al. (14), confirming their close relationship. Figure 1 shows the genome organization of PcMV.

Analysis of the PcMV ORFs. The coding region of PcMV ORF1 extends from nucleotide 158 to 5815 and encodes a putative polypeptide consisting of 1,886 aa residues $(216.3 \mathrm{kDa})$. This protein contains motifs associated with the methyltransferase domain (27), the helicase domain (8), and the RNA-dependent RNA polymerase domain (20), and likely represents the replicase region of the genome (Fig. 1). The deduced amino acid sequence of PcMV ORF1 most closely matches the corresponding ORF1 of CMLV (76\% identity), isolates of Apple chlorotic leaf spot virus (ACLSV, 60\% identity) and Apricot pseudo-chlorotic leaf spot virus (ApCLSV, 59\% identity). The replicase protein of PcMV, CMLV, ACLSV, and ApCLSV are of similar sizes with a molecular mass of approximately $216 \mathrm{kDa}$. Within the amino acid sequence of PcMV ORF1, there is a cysteine and a histidine residue at positions 855 and 931, respectively. These occur in a context similar to that of the cysteine and histidine residues identified by German-Retana et al. (6) in association with ACLSV. They may or may not be representative of papain-like protease activity (6), hence the question marks in Figure 1.

ORF2 overlaps ORF1 and extends from an AUG codon at nucleotide 5736 to 5738 , to a stop codon at position 7002 to 7004 , resulting in a putative protein consisting of 422 aa $(47.2 \mathrm{kDa})$. Alignment of the protein reveals $72 \%$ identity with the ORF2 sequence of CMLV (47-kDa protein), $47 \%$ identity with the $51-\mathrm{kDa}$ protein of ApCLSV, and 45 to $47 \%$ identity with the $50-\mathrm{kDa}$ protein of isolates of ACLSV. The 50-kDa protein encoded by ORF2 of ACLSV was associated with virus movement (5). Yoshikawa et al. (29) used green fluorescent protein labeling of the 50-kDa protein to confirm this function. It is likely, therefore, that the 47.2-kDa protein of PcMV is associated with cell-to-cell movement of the virus.

ORF3 overlaps ORF2 and extends from a start codon at nucleotide position 6724 to 6726 to nucleotide 7302 encoding a protein consisting of 193 aa $(21.7 \mathrm{kDa})$. This protein has $83 \%, 53$ to $55 \%$, and $54 \%$ identity compared with the CP of CMLV, isolates of ACLSV, and ApCLSV, respectively. The CP of PcMV is of identical molecular mass compared with the CP of CMLV, estimated at $20.5 \mathrm{kDa}$ by western blot analysis (13). The CP size based on the deduced amino acid sequence of ORF3 is estimated at $21.7 \mathrm{kDa}$, which is similar to that determined by the electrophoretic mobility of the protein. The CPs of all four available isolates of PcMV (Table 1) were sequenced and the deduced amino acid sequences were compared (data not shown). The percent identity of the $\mathrm{CP}$

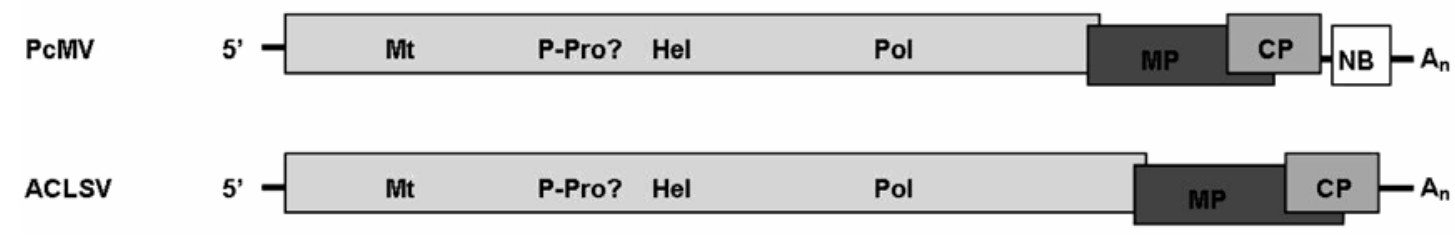

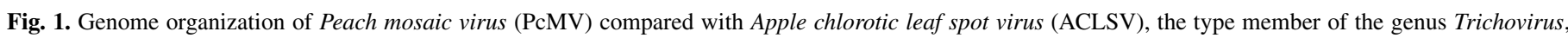

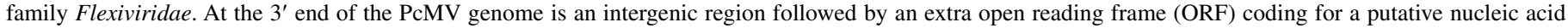

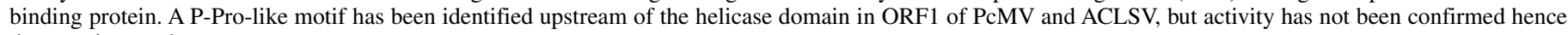
the question mark.

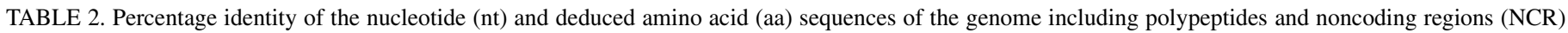
of Peach mosaic virus compared with Cherry mottle leaf virus ${ }^{\mathrm{a}}$

\begin{tabular}{|c|c|c|c|c|c|c|c|}
\hline Complete genome (nt) & 5'NCR (nt) & ORF1 (aa) & ORF2 (aa) & ORF3 (aa) & IG-NCR (nt) & ORF4 (aa) & $3^{\prime} \mathrm{NCR}$ (nt) \\
\hline 73 & 74 & 77 & 73 & 83 & 51 & 54 & 86 \\
\hline
\end{tabular}

a $\mathrm{ORF}=$ open reading frame; $\mathrm{IG}-\mathrm{NCR}=$ intergenic $\mathrm{NCR}$. 
sequences of isolates of PcMV varied from 97 to $99 \%$ compared with $83 \%$ identity for the CP of CMLV. Variability among PcMV isolates was confined mainly within the region of amino acid residues 10 to 32 at the $\mathrm{N}$ terminus of the $\mathrm{CP}$. Despite the relatively low identity between the $\mathrm{CP}$ amino acid sequence of PcMV and CMLV, conserved regions were identified that likely account for the serological cross reactivity observed.

ORF4 is separated from ORF3 by a 148-nt noncoding fragment, and extends from nucleotide 7451 to 7861 encoding a putative protein consisting of 137 aa $(15.7 \mathrm{kDa})$. BLAST searches with this region of the genome produced matches with the corresponding protein of CMLV, at $54 \%$ identity. The nucleic acidbinding (NB) protein motif described by James et al. (14) was identified between position 56 to 77 of the 137 aa protein. This motif is conserved across several genera within the family Flexiviridae (Fig. 2). Except for CMLV, no matches were found with any other members of the genus Trichovirus, all of which lack an ORF4. Matches of regions encompassing the putative NB protein motif were observed for members of the genera Allexivirus, Carlavirus, and the genus Vitivirus (Fig. 2). The polypeptide encoded by ORF4 contains the arginine-rich and zinc finger motifs typical of the NB protein (4). The zinc finger motif is similar to that described for the carlaviruses Potato virus $M$, Potato virus $S$, and Lily symptomless virus (9). There are four cysteine residues in the midregion of the amino acid sequence of the ORF4 protein of PcMV and CMLV, at amino acid positions 17, 20, 25, and 33 (Fig. 2). These appear to be in a suitable context and may have the potential for forming a zinc finger motif similar to that described for the carlaviruses Potato virus $M$, Potato virus $S$, and Lily symptomless virus (9). This differs from the C-X2-C-X4-H-X4-C zinc finger motif described by Green and Berg (10) and associated with members of the genus Vitivirus (4). The histidine residue prominent in the zinc finger of the genus Vitivirus is absent from members of the genus Carlavirus, and from PcMV and CMLV. Three of these cysteine residues (Fig. 2, positions 72, 75, and 86) are conserved across all viruses in this figure, including members from the genera Allexivirus, Carlavirus, Vitivirus, and NB protein-containing members of the genus Trichovirus.

Analysis of PcMV NCRs. Three NCRs were identified as components of the genome of PcMV: (i) a 5' NCR consisting of 157 nts, (ii) a $3^{\prime}$ NCR consisting of 127 nts, and (iii) an intergenic (IG)-NCR between ORF3 and ORF4 consisting of 148 nts. The highest level of identity was observed between the NCRs of PcMV and CMLV. Identity of the 5' NCR was estimated at $74 \%$, with a 110-nt fragment in this region having 92\% identity (nucleotide 1 to 110 for PcMV, and 22 to 131 for CMLV). Analysis and comparison of the three NCRs of PcMV and CMLV re- vealed that the $3^{\prime} \mathrm{NCR}$ has the highest level of identity at $86 \%$ (Table 2). PcMV and CMLV have a similar genomic organization, both containing an IG-NCR between ORF3 and ORF4, a region that shares only modest levels of identity at $51 \%$. No other matches of any significance were identified after BLAST searches.

Phylogenetic analysis. Phylogenetic analyses were carried out to determine the genetic relationships of PcMV to CMLV and other members of the family Flexiviridae. In analyses using the complete genome sequence (nucleotides), the amino acid sequence of the replicase region (ORF1), and the CP amino acid sequence (ORF3), PcMV grouped consistently with members of the genus Trichovirus. Phylogenetic analysis based on the CP amino acid sequence, with Strawberry mild yellow edge virus used as an outgroup, is shown in Figure 3. PcMV and CMLV formed a separate minor group distinct from ACLSV, ApCLSV, and GINV. PcMV is closely related, but distinct from CMLV. The high bootstrap values validate the tree topology and relationships observed (Fig. 3).

Phylogenetic analysis was attempted using the amino acid sequence of ORF4 of PcMV. The only relationships observed, based on limited sequence identity, were with CMLV and members of the genera Allexivirus, Carlavirus, and Vitivirus. Sequence identity was observed mainly in a region encompassing the putative NB protein domain (Fig. 2). This region of the NB protein was used in phylogenetic analysis to determine the relationships of the PcMV NB protein to other viruses containing this protein. PcMV and CMLV grouped together and formed a group separate from members of other genera (Fig. 4).

Detection of the expressed PcMV CP. The His-Tag facilitated purification of the expressed protein by Ni-NTA agarose column affinity chromatography. The validity of the ORF identified as encoding the CP of PcMV was supported by western blot detection of the purified fusion protein, using MAb 1162-A2 (Fig. 5, lane 3 ). The expressed fusion protein consisted of the PcMV CP, the TEE, the His-Tag, and Factor Xa site; consequently, it displayed slower electrophoretic mobility than the native virus CP (Fig. 5, lane 2).

PcMV-specific RT-PCR. A variety of fruit trees infected with a range of viruses were screened by RT-PCR(A) using primers PM16AFF and PM-16AFR (Table 1). Positive RT-PCR reactions were observed with certain infected Prunus plants that were PcMV negative when screened by biological indexing on the woody indicator 'Elberta' peach (Table 1). Also, an infected apple accession tested positive (Table 1). Apple is not known as a host for PcMV. These positive tests included accessions 1288-4, 98211P5, and 996-1A (Table 1; Fig. 6A). To confirm that cross reactions were occurring and not misdiagnosis, the amplified fragments were cloned, sequenced, and found to be 95 to $99 \%$

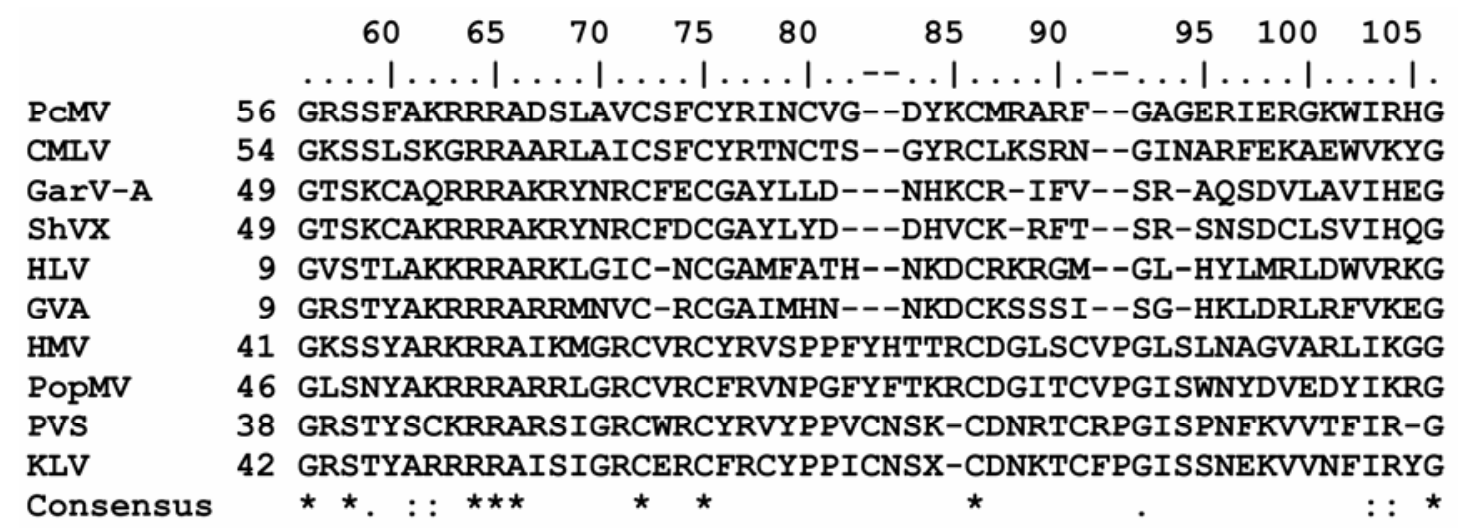

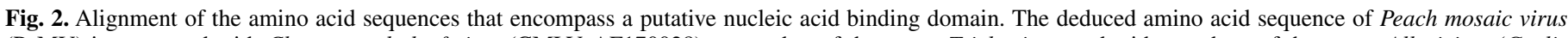

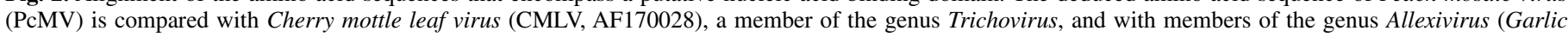

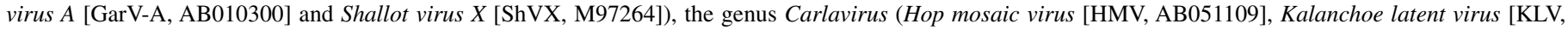

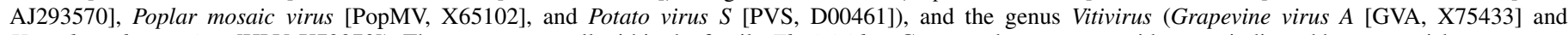
Heracleum latent virus [HLV, X79270]). These genera are all within the family Flexiviridae. Conserved consensus residues are indicated by an asterisk. 
identical to isolates of ACLSV (data not shown). A similar group of infected plants was screened with the primers PM-AF1 and PM-AFR (RT-PCR(B)), including accessions 1288-4, 982-11P5, and 996-1A. No cross reactions were observed with any of the ACLSV isolates, or with any other virus included in the study (Table 1; Fig. 6B). The primers amplified the expected 383-bp fragment with all available isolates of PcMV (Fig. 6B, lanes 1 to 5). Primers PM-16AFF and PM-16AFR detected all isolates of PcMV (Fig. 6A), but cross reactions with certain ACLSV isolates limit their usefulness for routine diagnosis. The reverse primer PM-16AFR is downstream of PM-AFR. It is possible, therefore, to do a seminested RT-PCR using cDNA generated by PM16AFR for amplification with the PM-AF1 and PM-AFR primers. This has the potential for an increase in sensitivity and specificity of the test. The PcMV-specific fragments shown in Figure 6B were amplified using seminested RT-PCR.

\section{DISCUSSION}

PcMV is serologically related to CMLV, and both viruses have $\mathrm{CP}$ subunits with identical electrophoretic mobility $(7,13,25)$.
However, PcMV and CMLV cause distinct diseases, have different host ranges, and are vectored by different eriophyid mites $(13,19,23,24)$. One objective of this study was to determine, by genome analysis and comparison, whether these are strains of the same virus, or closely related but distinct viruses. Both viruses have identical genome organization with four ORFs and three NCRs. The percent identity is less than $85 \%$ for every region of the genome (54 to $83 \%$ ), except the $3^{\prime} \mathrm{NCR}$ at $86 \%$. Comparison of the nucleotide sequence of the entire genome of PcMV and CMLV produced $73 \%$ identity. The CP amino acid sequences of isolates of PcMV have identity ranging from 97 to $99 \%$ compared with $83 \%$ for CMLV. The data indicates that although these viruses are closely related, PcMV and CMLV represent distinct viruses.

To assist in validating the genomic organization and putative ORFs proposed, PcMV CP was expressed as a fusion protein and detected using MAb 1162-A2. MAb 1162-A2 was produced using purified CMLV (15). CMLV and PcMV are serologically related and the MAb reliably detects both CMLV and PcMV $(7,13,25)$. Reaction of the virus-specific MAb 1162-A2 with the expressed fusion protein, that includes the protein encoded by the putative

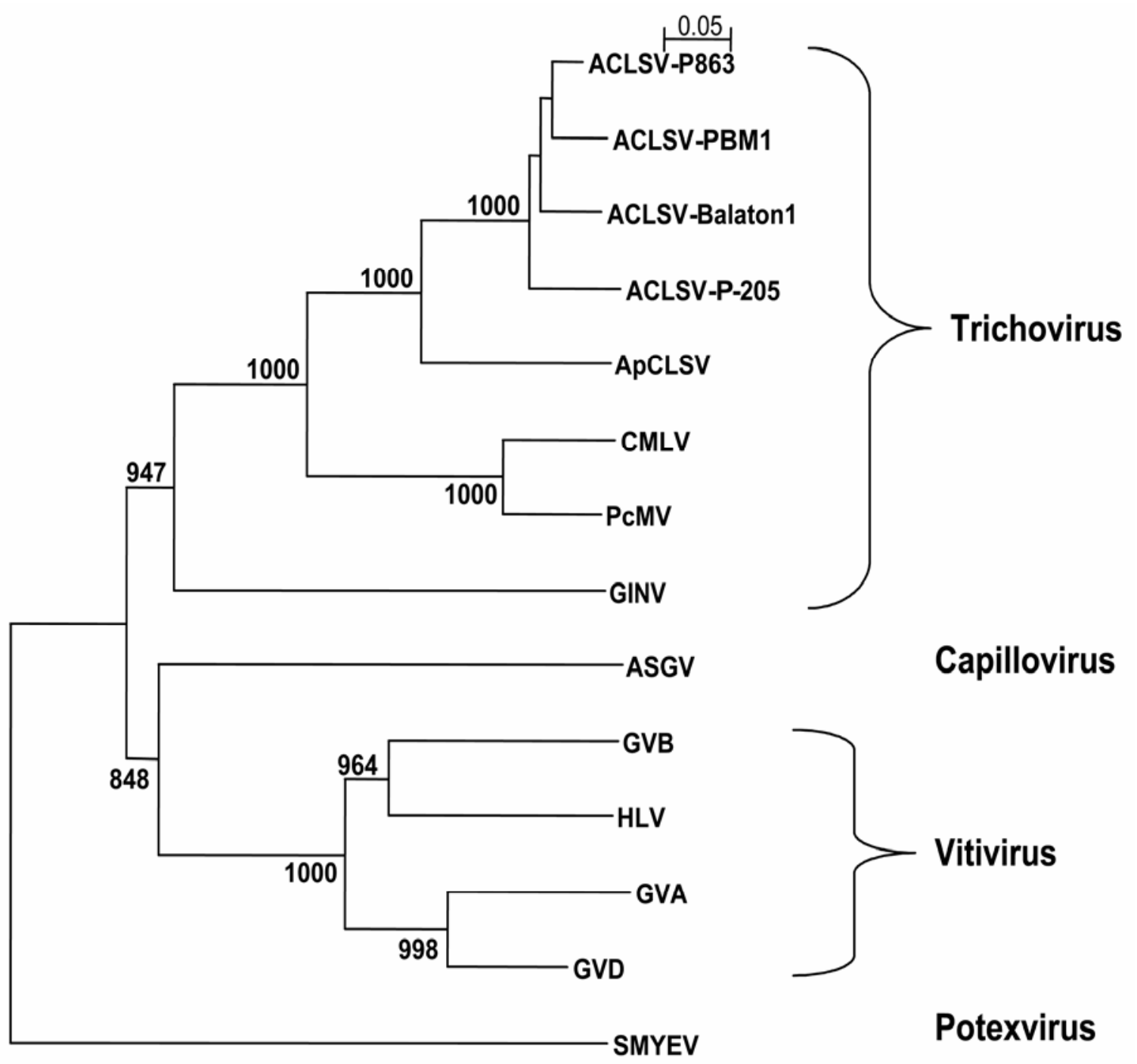

Fig. 3. Phylogenetic relationships of Peach mosaic virus (PcMV) and members of genera within the family Flexiviridae based on the amino acid sequences of the coat protein. Virus isolates with accession numbers include Apple chlorotic leaf spot virus P863 (ACLSV, M58152), ACLSV PBM1 (CAB46653), ACLSV Balaton1 (CAA68082), ACLSV P205 (BAA03643), Apricot pseudo-chlorotic leaf spot virus (ApCLSV, AAW55586), Apple stem grooving virus (ASGV, D14995), Cherry mottle leaf virus (CMLV, AF170028), Grapevine berry inner necrosis virus (GINV, D88448), Grapevine virus A (GVA, AF441235), Grapevine virus B (GVB, X75448), Grapevine virus D (GVD, Y07764), Heracleum latent virus (HLV, X79270), and Strawberry mild yellow edge virus (SMYEV, D12517) as an outgroup. The tree was produced using the N-J Tree option of Clustal-X (28). Bootstrap values out of 1,000 replicates are shown, and the scale bar indicates the number of substitutions per residue. 


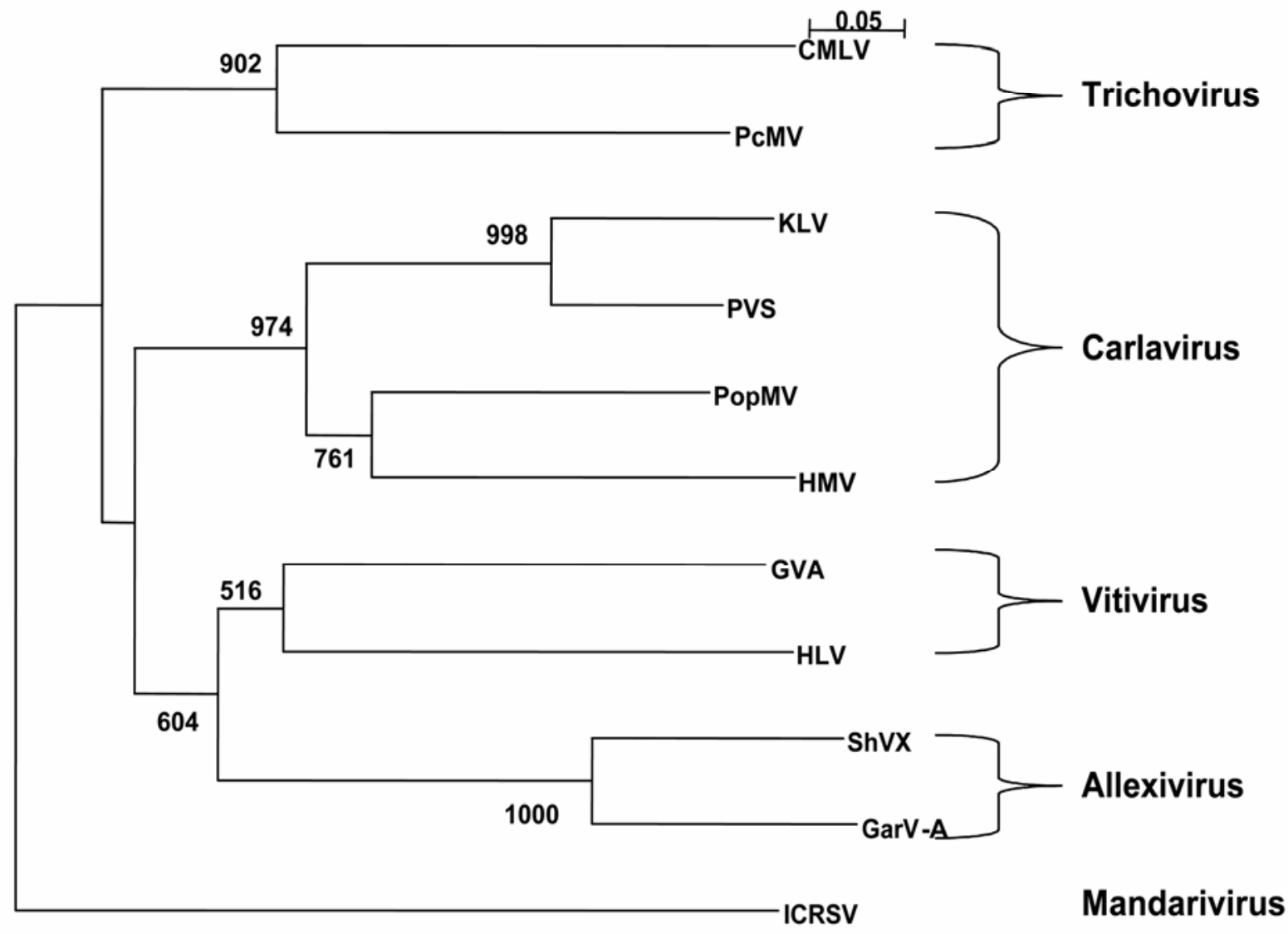

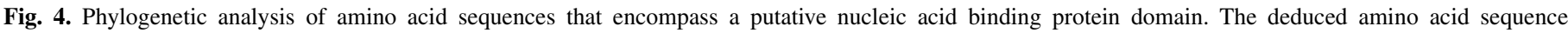

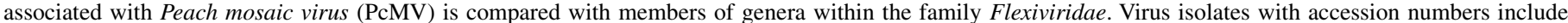

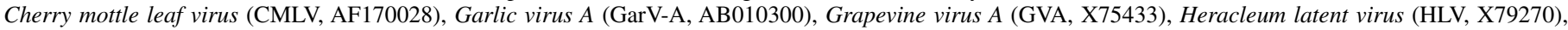

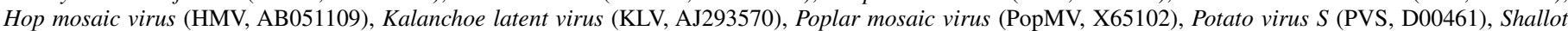

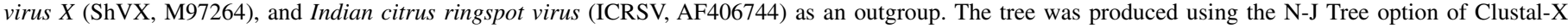
(28). Bootstrap values out of 1,000 replicates are shown, and the scale bar indicates the number of substitutions per residue.

CP ORF, confirms that PcMV ORF3 does indeed encode the PcMV CP. This supports the genome organization proposed.

Phylogenetic analyses based on the entire genome sequence (nucleotides), the amino acid sequence of the replicase region (ORF1), and the CP region (ORF3) support classification of PcMV, along with CMLV, as members of the genus Trichovirus. The genome organization of PcMV and CMLV is identical to members of the genus Trichovirus, except for an IG-NCR and a fourth ORF present at the $3^{\prime}$ end of the genome of PcMV and CMLV. These are not present in other members of the genus Trichovirus. ORF4 of PcMV and CMLV contain a putative NB protein motif (14). A region encompassing this motif displays identity with the NB protein of members of the genera Allexivirus, Carlavirus, and Vitivirus. These, including the genus Trichovirus, are all members of the family Flexiviridae. PcMV and CMLV appear to be products of a recombination event between members of the genus Trichovirus and members of an NB protein-containing group of viruses likely within the family Flexiviridae. The low identity levels preclude accurate determination of the origin of the putative NB protein of PcMV and CMLV. The putative NB protein is highly variable, both in size and sequence, with relatively low levels of identity even between members of the same genus, e.g., 54\% identity for the putative NB protein of PcMV compared with that of CMLV. The divergence observed may indicate an ancient recombination event or a high rate of mutation in this region. Another possible explanation is that members of the genus Trichovirus, such as ACLSV, may have been subjected to a deletion event resulting in the loss of ORF4. This is a real possibility since the NB protein encoded by ORF4 does not appear essential for virus replication and survival as demonstrated by ACLSV and GINV, viable members of the genus Trichovirus. The configuration of the putative zinc finger motif of the ORF4 of PcMV and CMLV suggests that, at least in this region, they are more closely related to the genus Carlavirus than to the genus Vitivirus. However, the lack of homology of the IG-NCR may suggest an origin other than any of the genera identified above. It is rather interesting that, in general, within the family Flexiviridae, members of genera containing the NB protein are insect transmitted (Carlavirus, Allexivirus, and Vitivirus), while members of genera lacking this protein (Potexvirus, Foveavirus, and Capillovirus) are not insect transmitted (1). It is tempting to speculate that the NB protein may be associated with insect transmission. This appears to be contradicted by the fact that GINV of the genus Trichovirus lacks the NB protein but is transmitted by mites. Also, as of yet no insect vector has been identified for the NB protein-containing Indian citrus ringspot virus, the single member of the genus Mandarivirus, family Flexiviridae (1). Galiakparov et al. (4) has shown that amino acid mutations in the NB protein influences symptom development and expression of the movement protein. It is conceivable, therefore, that the presence of ORF4 encoding a putative NB protein may confer unique biological traits to PcMV and CMLV. This region of the genome may influence several viral functions but much more research is needed to elucidate the role(s) of this putative protein.

There are sections of the RNA-dependent RNA polymerase region that are conserved across member genera of the family Flexiviridae, facilitating the development of broad spectrum tests (3). Therefore, cross reactivity of PcMV-derived primers targeting this 


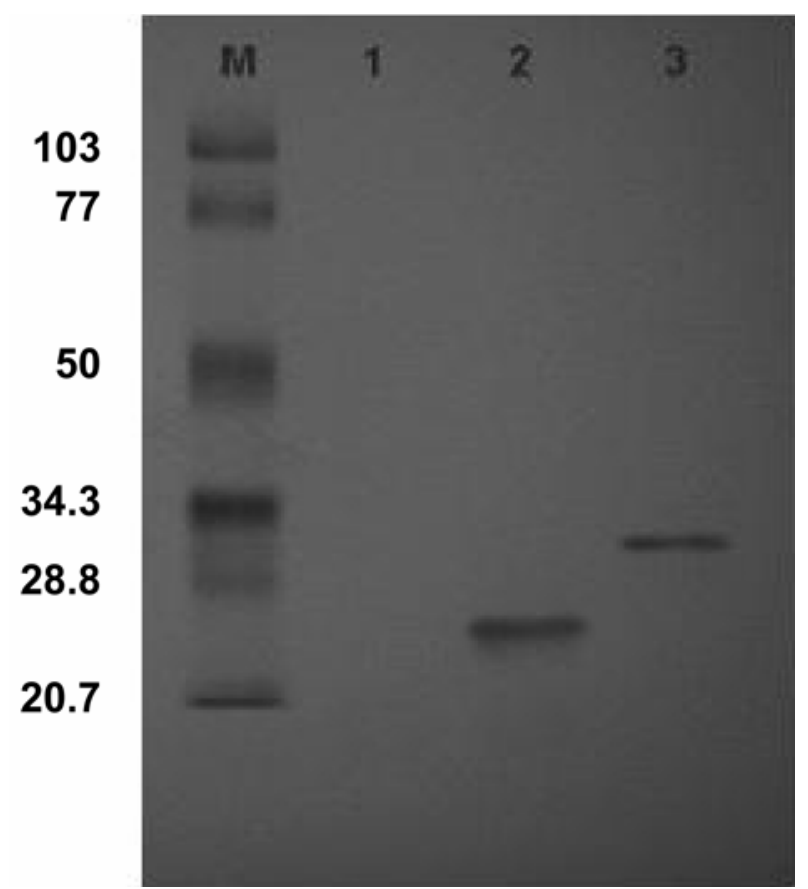

Fig. 5. Western blot detection of a fusion protein that includes the coat protein of Peach mosaic virus (PcMV) expressed in Escherichia coli BL21 (DE3). The Cherry mottle leaf virus derived monoclonal antibody 1162-A2, which cross reacts with PcMV, was used for detection. Lane M, Bio-Rad prestained sodium dodecyl sulfate-polyacrylamide gel electrophoresis standards, low range (kDa); lane 1, healthy Chenopodium quinoa; lane 2, C. quinoa infected with PcMV; and lane 3, expressed fusion protein from E. coli cells transformed with $\mathrm{pCOLD/PcM-CP}$ (expression vector containing PcMV CP gene).

region with ACLSV is not surprising. This phenomenon should be considered in the development of diagnostic tests (serology and nucleic acid-based) for members of this family. In this study, a range of viruses representing different genera within that family were screened for cross reactivity. Primers PM-AF1 and PM-AFR were specific and able to detect all available isolates of PcMV. No cross reactions were observed with any of the other viruses, confirming the specificity of this test. This test will be of value for stone fruit virus certification since accurate identification of viruses of economic and quarantine significance allows effective monitoring and control of these viruses.

In summary, PcMV and CMLV are distinct viruses that appear to be the products of recombination. The organization of these viruses is identical to that of members of the genus Trichovirus, except for the addition of an extra ORF (ORF4) separated by an IGNCR at the $3^{\prime}$ end of the genome. It is possible also that PcMV and CMLV represent the true genome organization of this group and that members such as ACLSV are the products of a deletion event. Phylogenetic analysis of the genome of PcMV supports classification of this virus as a member of the genus Trichovirus. However, the presence of an extra ORF with some identity to the NB-containing ORF in other genera of the family Flexiviridae cannot be ignored. The acquisition or loss of individual genes may not justify removing a virus from a particular genus, especially if analysis of every other region of the genome supports existing classification. As more and more sequence data become available, classification may be modified and/or refined to reflect subgroups with unique features within the genus Trichovirus, or it may become necessary to create a new genus.

\section{ACKNOWLEDGMENTS}

We thank W. E. Howell (Prosser) and H. J. Larsen (Colorado State University) for providing the isolates of Peach mosaic virus used in this study.
A

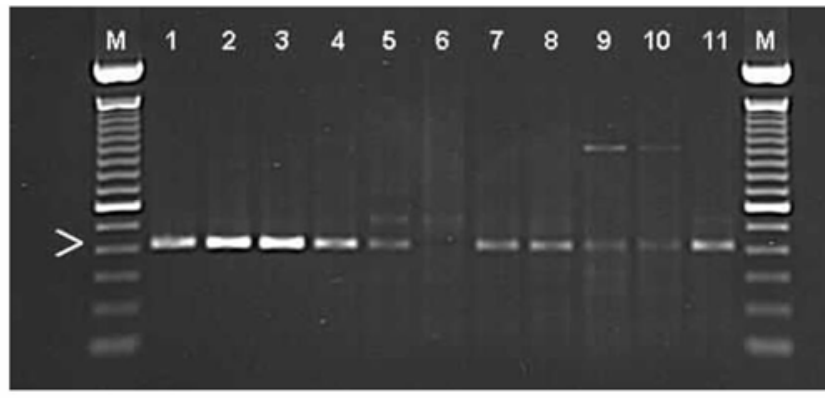

B

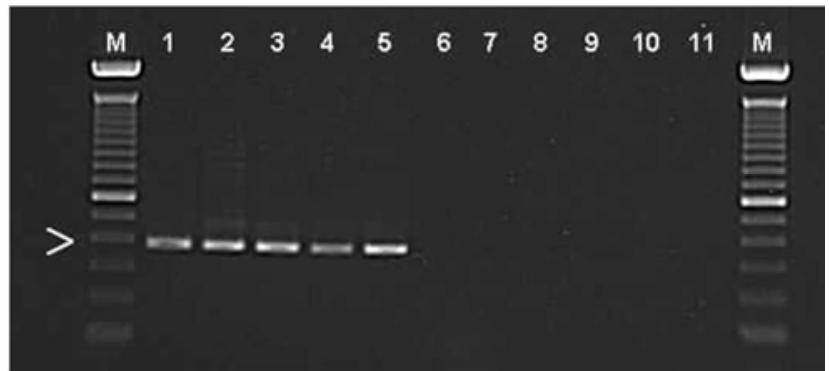

Fig. 6. Reverse transcription-polymerase chain reaction (RT-PCR) analysis comparing the specificity of Peach mosaic virus (PcMV)-derived primers. A shows the results using primer set A (PM16AFF/PM16AFR) with amplification of a 419-bp fragment. B shows the results using primer set B (PMAF1/PMAFR) in a seminested PCR, with amplification of a 383-bp fragment. Lane M, 100-bp DNA ladder; lane 1, PcMV CA1 in peach; lane 2, another accession of PcMV CA1 in peach; lane 3, PcMV CA2 in peach; lane 4, PcMV CA3 in peach; lane 5, PcMV CL2 in peach; lane 6, healthy peach; lane 7, Apple chlorotic leaf spot virus (ACLSV) isolate 1288-4 in peach; lane 8, ACLSV isolate 996-1A in plum; lane 9, ACLSV isolate 982-11P5 in apple; lane 10, another sample of ACLSV isolate 982-11P5 in apple; and lane 11, another sample of ACLSV isolate 1288-4 in peach.

\section{LITERATURE CITED}

1. Adams, M. J., Antoniw, J. F., Bar-Joseph, M., Brunt, A. A., Candresse, T., Foster, G. D., Martelli, G. P., Milne, R. G., and Fauquet, C. M. 2004. The new plant virus family Flexiviridae and assessment of molecular criteria for species demarcation. Arch. Virol. 149:1045-1060.

2. Bodine, E. W. 1934. Occurrence of peach mosaic in Colorado. Plant Dis. Rep. 18:132.

3. Foissac, X., Svanella-Dumas, L., Dulucq, M. J., Candresse, T., and Gentit, P. 2001. Polyvalent detection of fruit tree tricho, capillo, and foveaviruses by nested RT-PCR using degenerated and inosine containing primers (PDO RT-PCR). Acta Hort. 550:37-43.

4. Galiakparov, N., Tanne, E., Mawassi, M., Gafny, R., and Sela, I. 2003. ORF5 of Grapevine virus $A$ encodes a nucleic acid-binding protein and affects pathogenesis. Virus Genes 27:257-262.

5. German, S., Candresse, T., Lanneau, M., Huet, J. C., Pernollet, J. C., and Dunez, J. 1990. Nucleotide sequence and genomic organization of apple chlorotic leaf spot closterovirus. Virology 179:104-112.

6. German-Retana, S., Bergey, B., Delbos, R. P., Candresse, T., and Dunez, J. 1997. Complete nucleotide sequence of the genome of a severe cherry isolate of apple chlorotic leaf spot trichovirus (ACLSV). Arch. Virol. 142:833-841.

7. Gispert, C., Perring, T. M., and Creamer, R. 1998. Purification and characterization of peach mosaic virus. Plant Dis. 82:905-908.

8. Gorbalenya, A. E., and Koonin, E. V. 1989. Viral proteins containing the purine-NTP sequence pattern. Nucleic Acids Res. 17:8413-8440.

9. Gramstat, A., Courtpozanis, A., and Rohde, W. 1990. The $12 \mathrm{kDa}$ protein of potato virus $\mathrm{M}$ displays properties of a nucleic acid-binding regulatory protein. FEBS Letts. 276:34-38.

10. Green, L. M., and Berg, J. M. 1989. A retroviral Cys-Xaa 2 -Cys-Xaa-His$\mathrm{Xaa}_{4}$-Cys peptide binds metal ions: Spectroscopic studies and a proposed three-dimensional structure. PNAS 86:4047-4051.

11. Hutchins, L. M. 1932. Peach mosaic-a new virus disease. Science $76: 123$.

12. James, D., and Howell, W. E. 1993. Comparisons of cherry mottle leaf virus and a virus associated with peach mosaic disease. Page 304 in: Proc. 6th International Congress of Plant Pathology (Abstr.), Montreal, Canada.

13. James, D., and Howell, W. E. 1998. Isolation and partial characterization of a filamentous virus associated with peach mosaic disease. Plant Dis. 82:909-913 
14. James, D., Jelkmann, W., and Upton, C. 2000. Nucleotide sequence and genome organization of cherry mottle leaf virus and its relationship to members of the Trichovirus genus. Arch. Virol. 145:995-1007.

15. James, D., and Mukerji, S. 1993. Mechanical transmission, identification, and characterization of a virus associated with mottle leaf in cherry. Plant Dis. 77:271-275.

16. James, D., and Upton, C. 1999. Single primer pair designs that facilitate simultaneous detection and differentiation of peach mosaic virus and cherry mottle leaf virus. J. Virol. Methods 83:103-111.

17. James, D., and Varga, A. 2005. Nucleotide sequence analysis of Plum pox virus isolate W3174: Evidence of a new strain. Virus Res. 110:143-150.

18. James, D., Varga, A., Thompson, D., and Hayes, S. 2003. Detection of a new and unusual isolate of Plum pox virus in plum (Prunus domestica). Plant Dis. 87:1119-1124.

19. Keifer, H. H., and Wilson, N. S. 1955. A new species of eriophyid mite responsible for the vection of peach mosaic virus. Bull. Calif. Dep. Agric. 44:145-146.

20. Koonin, E. V. 1991. The phylogeny of RNA-dependent RNA polymerases of positive-strand RNA viruses. J. Gen. Virol. 72:2197-2206.

21. Larsen, H. J., and Oldfield, G. N. 1995. Peach mosaic. Pages 67-68 in: Compendium of Stone Fruit Diseases. J. M. Ogawa, E. I. Zehr, G. W. Bird, D. F. Ritchie, K. Uriu, and J. K. Uyemoto, eds. The American Phytopathological Society, St. Paul, MN.

22. Morris, T. J., and Dodds, J. A. 1979. Isolation and analysis of doublestranded RNA from virus-infected plant and fungal tissue. Phytopathol- ogy 69:854-858.

23. Nemeth, M. 1986. Virus, Mycoplasma and Rickettsia Diseases of Fruit Trees. Akademiai Kiado, Budapest.

24. Oldfield, G. N. 1970. Mite transmission of plant viruses. Annu. Rev. Entomol. 15:343-380.

25. Oldfield, G. N., Creamer, R., Gispert, C., Osorio, F., Rodriguez, R., and Perring, T. M. 1995. Incidence and distribution of peach mosaic and its vector, Eriophyes insidiosus (Acari: Eriophyidae) in Mexico. Plant Dis. 79:186-189.

26. Pine, T. S. 1976. Peach mosaic. Pages 61-70 in: Virus Diseases and Noninfectious Disorders of Stone Fruits in North America. R. M. Gilmer, J. D. Moore, G. Nyland, M. F. Welsh, and T. S. Pine, eds. U.S. Dep. Agric. Handbook No. 437

27. Rozanov, M. N., Koonin, E. V., and Gorbalenya, A. E. 1992. Conservation of the putative methyltransferase domain: A hallmark of the 'Sindbislike' supergroup of positive-strand RNA viruses. J. Gen. Virol. 73:21292134.

28. Thompson, J. D., Gibson, T. J., Plewniak, F., Jeanmougin, F., and Higgins, D. G. 1997. The ClustalX windows interface: Flexible strategies for multiple sequence alignment aided by quality analysis tools. Nucleic Acids Res. 24:4876-4882.

29. Yoshikawa, N., Oogake, S., Terada, M., Miyabayashi, S., Ikeda, Y., Takahashi, T., and Ogawa, T. 1999. Apple chlorotic leaf spot virus $50 \mathrm{kDa}$ protein is targeted to plasmodesmata and accumulates in sieve elements in transgenic plant leaves. Arch. Virol. 144:2475-2483. 